Palavras chave: Manejo florestal sustentado Araucária Competição florestal

Histórico:

Recebido I | $/ 05 / 201$ I

Aceito 29/04/2014

Keywords: Sustainable forest management Floresta ombrófila mista Forest competition

Correspondência: hessandre@yahoo.com.br
André Felipe Hess', Myrcia Minatti', Linamara Ferrari', Benhur Ayrton Pintro'

\section{MANEJO DE FLORESTA OMBRÓFILA MISTA PELO MÉTODO DE LIOCOURT, MUNICÍPIO DE PAINEL, SC}

RESUMO: Objetivou-se, neste trabalho, conhecer a distribuição diamétrica e calcular o valor do quociente ' $q$ ' de Liocourt da espécie Araucaria angustifolia (Bertol.) Kuntze em floresta natural, bem como avaliar os índices de competição em um fragmento com área de 74,2 ha. Para tanto, foi utilizado o método de área fixa com parcelas permanentes de $400 \mathrm{~m}^{2}$, totalizando um hectare de área amostral. Em todas as parcelas, foram medidos os indivíduos com DAP $\geq$ a $10 \mathrm{~cm}$ e, posteriormente, distribuídos em classe diamétrica para ajuste da função de probabilidade de densidade e determinação de índice de competição. A floresta apresentou uma densidade de 228 árvores por hectare e área basal de $19,49 \mathrm{~m}^{2} \cdot \mathrm{ha}^{-1}$. O valor do quociente 'q' para a floresta foi de I,I, indicando que as taxas de mortalidade e recrutamento encontram-se em equilíbrio. Os índices de competição calculados indicaram que a floresta apresenta-se em estágio de competição.

\section{ARAUCARIA FOREST MANAGEMENT BY THE LIOCOURT METHOD, PAINEL MUNICIPALITY, SC}

ABSTRACT: The aim of this study was to describe the diameter distribution and calculate the value of the Liocourt quotient ' $q$ ' for the species Araucaria angustifolia (Bertol.) Kuntze in natural forest, as well as evaluating competition indexes in a fragment with an area of 74.2 ha. Fixed area sample plots of $400 \mathrm{~m}^{2}$ were used in this study, totaling one hectare sampling area. Trees with $\mathrm{DBH} \geq 10 \mathrm{~cm}$ were measured in all plots and later distributed in diameter classes for the calculation of the density probability function and competition index. The forest showed a density of 228 trees per hectare and basal area of $19.49 \mathrm{~m}^{2}$.ha". . The value of the "q" quotient for the forest was I.I, indicating that the recruitment and mortality rates are in equilibrium. The calculated competition indices showed that the forest is in a competition stage, with Glover and Holl index of 0.9798 and $0.7069 \mathrm{~m}^{2}$ for the $\mathrm{BAL}_{\text {mod }}$. 


\section{INTRODUÇÃO}

A Floresta Ombrófila Mista é caracterizada pela presença de Araucaria angustifolia (Bertol.) Kuntze que, por sua abundância, porte e copas corimbiformes imprime $\circ$ aspecto fitofisionômico próprio dessa formação. Ela ocorre, ainda, intercaladamente com áreas savânicas e estépicas, originando um sistema de mosaico que caracteriza grande parte da paisagem da região Sul do Brasil (AUBREVILLE, 1954; BACKES, 2002; HUECK, 1972; KLEIN, 1960).

Desde o início da colonização, essa floresta fo explorada, sem que houvesse qualquer preocupação com a sua preservação ou com a sustentabilidade dos processos extrativistas (HUECK, 1972). Os remanescentes ainda existentes são encontrados em locais de difícil acesso, em áreas particulares ou em Unidades de Conservação. A continuidade do sistema florestal em questão constitui, hoje, um dos maiores desafios para os programas de conservação.

Esses programas devem ser fundamentados no conhecimento da diversidade e da estrutura, capazes de estabelecer estratégias adequadas de manejo, visando, principalmente, à conservação e ao uso múltiplo sustentável dos recursos florestais (SONEGO et al., 2007). De acordo com Schaaf et al. (2006), recuperar, conservar e utilizar racionalmente os benefícios advindos da Floresta Ombrófila Mista constituem um grande desafio, o qual não se consegue apenas por meio da legislação.

Existe a necessidade de conhecer os atributos da floresta por meio do inventário florestal, para que se tenha suporte dos planos de manejo florestais sustentados. Nesse contexto, a distribuição diamétrica assume particular importância no levantamento da estrutura horizontal de uma floresta, por permitir caracterizar uma tipologia florestal e, também, por ser um potente indicador do estoque em crescimento das florestas, além de fornecer subsídios para a tomada de decisões e do planejamento do manejo a ser aplicado em determinada área (MACHADO et al., 2009).

No desenvolvimento dos planos de manejo, tanto de florestas equiâneas e inequiâneas, além da distribuição diamétrica, é importante estudar os índices de competição. Campos (1997) relata que os índices de competição variam de modelo para modelo, mas, de modo geral, está em função do tamanho da árvore em relação ao tamanho e/ou à distância das árvores competidoras. A competição é um dos fatores ecológicos mais importantes no manejo florestal, já que quase todas as intervenções estão relacionadas com a manipulação desse fator ou das condições que o afetam. Dessa forma, regular a competição existente num povoamento florestal é de suma importância para seu crescimento (SCHNEIDER, 1993).

Nesse contexto, objetivou-se, neste trabalho, avaliar a necessidade de intervenção de uma área de Araucaria angustifolia, por meio do método de Liocourt e de índices de competição.

\section{MATERIAL E MÉTODOS}

\section{Descrição do local de estudo}

O estudo foi realizado em uma propriedade rural, com predomínio de Araucaria angustifolia, localizada no município de Painel, SC. As coordenadas geográficas são $50^{\circ} 07^{\prime} 30.6^{\prime \prime} \mathrm{W}$ e $27^{\circ} 55^{\prime} 0 \mathrm{I} .5^{\prime \prime} \mathrm{S}$, da microrregião do Planalto Serrano com altitude de $1.215 \mathrm{~m}$.

O clima da região é classificado, segundo Köeppen, como $\mathrm{Cfb}$, mesotérmico úmido, com temperatura média entre 15 e $16^{\circ} \mathrm{C}$ e precipitação pluviométrica média anual entre I.200 a $1.900 \mathrm{~mm}$, com chuvas bem distribuídas durante o ano.

Os solos da região são associações de solos litólicos distróficos e eutróficos, cambissolo álico Tb, horizonte A proeminente, textura muito argilosa, relevo forte ondulado e ondulado, ambos fase pedregosa campo e floresta subtropical e afloramento rochosos, com uso predominante de pecuária extensiva (EMPRESA DE PESQUISA AGROPECUÁRIA E EXTENSÃO RURAL DE SANTA CATARINA - EPAGRI, 2002).

\section{Proposição do plano de manejo}

Para avaliação da estrutura diamétrica, foram utilizadas 25 parcelas temporárias de $400 \mathrm{~m}^{2}$, distribuídas, sistematicamente, para uma melhor varredura da floresta. Foram medidos todos os indivíduos com diâmetro a I,30 m do solo(DAP), a partir de $10 \mathrm{~cm}$. Procedida a coleta, os indivíduos foram divididos em classes diamétricas, com intervalo de classe de $5 \mathrm{~cm}$. Com esses dados, foi obtido o quociente ' $q$ ' de Liocourt (DE LIOCOURT, I898), para a avaliação da estrutura diamétrica e proposição de manejo, bem como os valores do índice de competição.

Determinadas as classes diamétricas, foram ajustados os dados de frequência por classe de diâmetro, a partir da função de distribuição de Meyer linearizada (equação I), sendo $Y_{i}=$ número de árvores por classe de diâmetro; $X_{i}=$ centro de classe de diâmetro; 
In = logaritmo neperiano; $\beta_{0}, \beta_{1}=$ parâmetros do modelo; $\varepsilon_{i}=$ erro aleatório. Seu ajuste foi verificado pelo critério do Coeficiente de determinação ajustado ( $R^{2}$ aj.) e erro padrão relativo.

$\ln \left(Y_{i}\right)=\beta_{0}+\beta_{1} \cdot X_{i}+\varepsilon_{i}$

Com base na função de distribuição ajustada, foi obtido o quociente ' $q$ ' intrínseco da vegetação por meio da equação 2 , sendo utilizada a razão entre as frequências de uma classe de diâmetro qualquer $\left(X_{i}\right)$ pela classe imediatamente acima $\left(\mathrm{X}_{\mathrm{i}+1}\right)$.

$$
q=\frac{e^{\left(\hat{\beta}_{0}+\hat{\beta}_{1} \cdot X_{i}\right)}}{e^{\left(\hat{\beta}_{0}+\hat{\beta}_{1} \cdot X_{i}+1\right)}}
$$

Após se obter o valor de ' $q$ ', pode-se gerar a curva da diferença entre as frequências (observada e estimada), recalculando-se o valor de $\hat{\beta}_{\mid}$(equação 3 ), indicando quais as classes de diâmetro que possuem superávit de árvores, podendo então ser removidas.

$$
\hat{\beta}_{\mathrm{I}}=\frac{\ln (q)}{X_{i}-X_{i+1}}
$$

Mediante experimentação, determinou-se o diâmetro que deve ser mantido na área, adotando-se o critério de madurez, isto é, a idade em que a árvore atinge o culmínio (assíntota) do crescimento ou condição de exploração. A característica desse diâmetro deve permitir que o futuro volume de remoção anual do povoamento resulte no máximo.

Segundo o conceito de De Liocourt (1898), as árvores das classes diamétricas grandes devem ser eliminadas, porque seu incremento está abaixo do ritmo das árvores das classes menores. Assim, com base na distribuição diamétrica, valor de incremento e condição de exploração para araucária, estipularam-se como diâmetros limites de manejo os valores de 40 e $45 \mathrm{~cm}$, correspondendo respectivamente a uma área basal remanescente de $10,09 \mathrm{~m}^{2} \cdot \mathrm{ha}^{-1}$ e $12,01 \mathrm{~m}^{2} \cdot \mathrm{ha}^{-1}$, efetuando-se a simulação da área basal e número de árvores que podem ser removidas para prover o ajuste na curva da distribuição de frequência da floresta.

\section{Índices de competição}

Para a estimativa do índice de competição entre as árvores adjacentes, foi utilizado o índice de Glover e Hool (PRODAN et al., 1997) e o índice de BAL modificado (Basal area larger). $O$ índice de Glover e Hool relaciona o diâmetro da árvore avaliada com o diâmetro médio das árvores vizinhas, dentro de uma área previamente estabelecida, conforme equação 4 , sendo di = diâmetro da árvore considerada, em centímetros; $\overline{\mathrm{d}}$ = diâmetro médio das árvores vizinhas da considerada, em centímetros, variando de acordo com o número de árvores na parcela. Então, quanto menor for o índice, maior será a concorrência sofrida pela árvore.

$\mathrm{I}_{\mathrm{GH}}=\frac{\mathrm{d}_{\mathrm{i}}^{2}}{\overline{\mathrm{d}}_{2}}$

O segundo índice busca quantificar a concorrência por espaço entre as árvores, considerando como competidoras aquelas com área basal maior que a árvore considerada. Assim, quanto maior o índice, maior a competição exercida sobre o indivíduo considerado (DELLA-FLORA, 200I), sendo calculado pela equação 5 , sendo $n=$ número de árvores com circunferência à altura do peito maior que a árvore-amostra no ponto amostral; g = área transversal, em metros quadrados.

$\mathrm{BAL}=\sum_{\mathrm{i}=1}^{\mathrm{n}} \mathrm{g}$

Os índices de competição foram calculados para verificar se ocorre competição na floresta, pois a espécie apresenta-se dispersamente distribuída e, em alguns casos, ocorrendo em pequenos grupos, bem como avaliar o impacto da exploração anterior nessa tipologia florestal.

\section{RESULTADOS E DISCUSSÃO}

A distribuição diamétrica do povoamento pode ser observada na Figura I. A frequência observada não apresentou a curva na forma de J-invertido, típica de florestas nativas. Isso pode ter ocorrido, por se tratar de um fragmento que sofreu intervenção com retirada de indivíduos, principalmente nas classes de 42,5 até 47,5 cm, sendo observado pelo maior número de árvores nas classes intermediárias $(20-30-35 \mathrm{~cm})$ e após na de 50 $\mathrm{cm}$. Com a retirada dos indivíduos, abre-se espaço para o crescimento em diâmetro.

A floresta apresentou 228 árvores por hectare e área basal de $19,49 \mathrm{~m}^{2} \cdot \mathrm{ha}^{-1}$, sendo a propriedade com menor número de indivíduos encontrada em dois anos de avaliação de fragmentos de floresta com araucária (HESS, 20I0). Essa baixa densidade deve-se ao fato de a área ter sido intensamente explorada, contudo, sem plano de manejo adequado. 


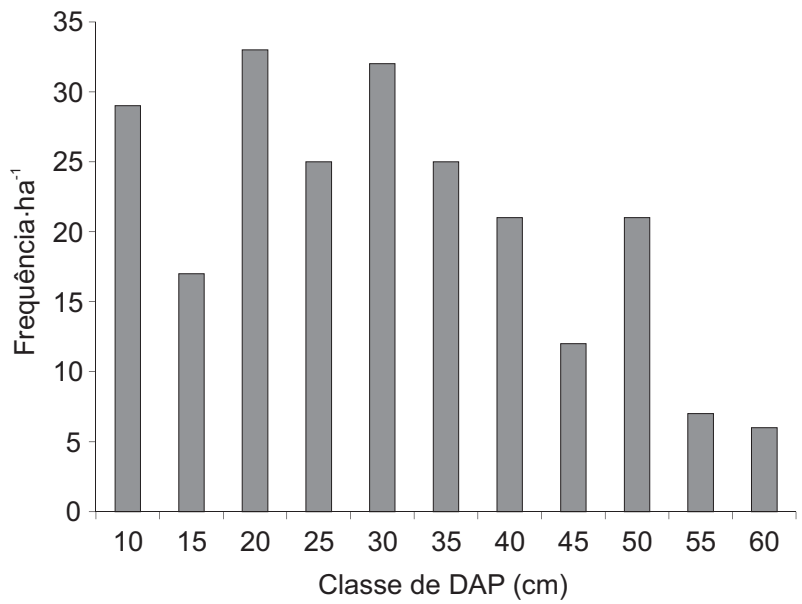

FIGURA I Frequência observada para Araucaria angustifolia, município de Painel, SC.

FIGURE I Observed frequently for Araucaria angustifolia, Painel municipality, SC.

Quando a espécie não apresenta distribuição diamétrica típica de J-invertido, caracteriza-se como falta de capacidade de regeneração e manutenção, bem como problemas de recrutamento e extração, pois existe um desbalanceamento entre as classes (NASCIMENTO et al., 2004).

Nesse sentido, Martins (199I) advertiu que a maior densidade de indivíduos menores não indica ausência de problemas de regeneração, devendo ser considerada com cautela, demonstrando a necessidade de uma análise mais detalhada, em nível específico e com um grupo maior de espécies para permitir interpretações mais seguras das distribuições diamétricas.

O valor do quociente de De Liocourt (I898) para a floresta estudada foi de I, I, e os coeficientes da equação de distribuição de frequência ajustada e seus parâmetros estatísticos foram, $\beta_{0}=3,83478 ; \beta_{1}=$ $-0,02668$; $R^{2}$ aj. de 0,52 ; erro padrão relativo de $1,97 \%$.

A maioria dos indivíduos concentrou-se na classe inicial $(10 \mathrm{~cm})$ e nas intermediarias $(20,25,30$ $\mathrm{cm}$ ), conforme Figura I. Como o valor do quociente é influenciado pelas frequências de todas as classes diamétricas e, não apenas das classes menores, as ausências de indivíduos de determinadas classes ou densidade menor nas maiores classes podem explicar o valor próximo a I de ' $q$ ', indicando que esse fragmento sofreu intervenções antrópicas pela sua estrutura não balanceada (ALVES JÚNIOR et al., 20I0; HESS, 20I0; NUNES et al., 2003).

Os coeficientes da função de Meyer foram recalculados e, para o diâmetro máximo remanescente de $40 \mathrm{~cm}$, foram $\beta_{0}=3,82743$ e $\beta_{1}=-0,01906$. Para o diâmetro máximo remanescente de $45 \mathrm{~cm}$, foram $\beta_{0}=3,73377$ e $\beta_{1}=-0,01906$. Pode-se, assim, definir a existência de classes superavitárias (árvores a remover) e deficitárias, as quais devem ter sua densidade aumentada, obtendo-se, assim, o número de árvores e a área basal a serem removidas de acordo com distribuição dos indivíduos em cada classe diamétrica conforme a Tabela le 2.

Conforme as Tabelas I e 2 e o regime de manejo estabelecido, poderão ser removidas árvores das classes de diâmetro $20,30,35,40$ e $50 \mathrm{~cm}$ para os diâmetros máximos desejados de 40 e $45 \mathrm{~cm}$. Representando 12 árvores por hectare e $1,17 \mathrm{~m}^{2} \cdot \mathrm{ha}^{-1}$ em área basal, totalizando $86,8 \mathrm{~m}^{2}$ total, para o diâmetro de $40 \mathrm{~cm}$ e, 22 árvores e 2, $18 \mathrm{~m}^{2} \cdot \mathrm{ha}^{-1}$ de área basal, com total de $161,76 \mathrm{~m}^{2}$ a ser removido para o diâmetro de $45 \mathrm{~cm}$, conferindo à floresta, em caso de cortes sucessivos, uma estrutura balanceada, pois há a tendência de aumento no crescimento e as árvores migrarem para classes de maior diâmetro, pois a retirada de indivíduos contribui para a regeneração da espécie, e diminuição da competição.

TABELA I Frequências observadas e estimadas, número de árvores e área basal a remover para o diâmetro máximo desejado de $40 \mathrm{~cm}$.

TABLE I Estimated and observed frequencies, tree quantities, and basal area for partial cutting to increase $\mathrm{DBH}$ to $40 \mathrm{~cm}$.

\begin{tabular}{ccccccc}
\hline cdap & FO/ha & $\begin{array}{c}\mathrm{G} \\
\left(\mathrm{m}^{2} / \mathrm{ha}\right)\end{array}$ & $\mathrm{FE} / \mathrm{ha}$ & $\mathrm{NR} / \mathrm{ha}$ & $\begin{array}{c}\mathrm{GR} \\
\left(\mathrm{m}^{2} / \mathrm{ha}\right)\end{array}$ & $\begin{array}{c}\mathrm{GRM} \\
\left(\mathrm{m}^{2} / \mathrm{ha}\right)\end{array}$ \\
\hline 10 & 29 & 0,2278 & 38 & - & - & 0,2278 \\
15 & 17 & 0,3004 & 35 & - & - & 0,3004 \\
20 & 33 & 1,0367 & 31 & 2 & 0,0628 & 0,9739 \\
25 & 25 & 1,2272 & 29 & - & - & 1,2272 \\
30 & 32 & 2,2619 & 26 & 6 & $0,424 \mathrm{I}$ & 1,8378 \\
35 & 25 & 2,4053 & 24 & 1 & 0,0962 & 2,3091 \\
40 & 21 & 2,6389 & 21 & - & - & 2,6389 \\
45 & 12 & 1,9085 & 19 & - & - & 1,9085 \\
50 & 21 & 4,1233 & 18 & 3 & 0,5890 & 3,5343 \\
55 & 7 & 1,6631 & 16 & - & - & 1,6631 \\
60 & 6 & 1,6965 & 15 & - & - & 1,6964 \\
\hline Total & 228 & 19,4897 & 271 & 12 & 1,1722 & 18,3174 \\
\hline
\end{tabular}

cdap= classe de diâmetro; $F O=$ frequência observada por hectare; $\mathrm{FE}=$ frequência estimada por hectare; $\mathrm{NR}=$ número de árvores a remover por hectare; $\mathrm{G}=$ área basal observada em metros quadrados por hectare; $G R=$ área basal a remover em metros quadrados por hectare; GRM = área basal remanescente por hectare. 
TABELA 2 Frequências observadas e estimadas, número de árvores e área basal a remover para o diâmetro máximo desejado de $45 \mathrm{~cm}$.

TABLE 2 Estimated and observed frequencies, tree quantities, and basal area for partial cutting to increase $\mathrm{DBH}$ to $45 \mathrm{~cm}$.

\begin{tabular}{ccccccc}
\hline cdap & FO/ha & $\begin{array}{c}\mathrm{G} \\
\left(\mathrm{m}^{2} / \mathrm{ha}\right)\end{array}$ & $\mathrm{FE} / \mathrm{ha}$ & $\mathrm{NR} / \mathrm{ha}$ & $\begin{array}{c}\mathrm{GR} \\
\left(\mathrm{m}^{2} / \mathrm{ha}\right)\end{array}$ & $\begin{array}{c}\mathrm{GRM} \\
\left(\mathrm{m}^{2} / \mathrm{ha}\right)\end{array}$ \\
\hline 10 & 29 & 0,2278 & 35 & - & - & 0,2278 \\
15 & 17 & 0,3004 & 31 & - & - & 0,3004 \\
20 & 33 & 1,0367 & 29 & 4 & 0,1257 & $0,911 \mathrm{I}$ \\
25 & 25 & 1,2272 & 26 & - & - & 1,2272 \\
30 & 32 & 2,2619 & 24 & 8 & 0,5655 & 1,6965 \\
35 & 25 & 2,4053 & 21 & 4 & 0,3848 & 2,0204 \\
40 & 21 & 2,6389 & 20 & 1 & - & 2,6389 \\
45 & 12 & 1,9085 & 18 & - & - & 1,9085 \\
50 & 21 & 4,1233 & 16 & 5 & 0,9817 & 3,1416 \\
55 & 7 & 1,6631 & 15 & - & - & 1,6631 \\
60 & 6 & 1,6965 & 13 & - & - & 1,6965 \\
\hline Total & 228 & 19,4897 & 247 & 22 & 2,0577 & 17,4319 \\
\hline
\end{tabular}

cdap = classe de diâmetro; $\mathrm{FO}=$ frequência observada por hectare; $\mathrm{FE}=$ frequência estimada por hectare; $\mathrm{NR}=$ número de árvores a remover por hectare; $\mathrm{G}$ = área basal observada em metros quadrados por hectare; $G R=$ área basal a remover em metros quadrados por hectare; GRM = área basal remanescente por hectare.

Esse baixo valor de área basal e número de árvores a retirar para as classes diamétricas, corroboram com a informação de intervenção passada nesse fragmento de floresta ombrófila mista que, segundo o proprietário, era explorada e a madeira utilizada para a indústria de palito de fósforo.

Para auxiliar na proposição de manejo pelo método de Liocourt, calcularam-se os índices de competição de Glover e Hool $\left(I_{G H}\right)$ e BAL modificado para as árvores amostra, considerando somente a espécie araucária, os quais são apresentados na (Tabela 3). Mesmo com densidade de 228 árvores.ha ${ }^{-1}$ o fragmento inventariado apresentou competição, necessitando regulação de densidade em algumas classes de diâmetro.

O índice de Glover e Holl $\left(\mathrm{I}_{G H}\right)$ calculado teve valor médio de 0,9798 , indicando uma competição entre os indivíduos dentro da parcela, isto é, há uma proximidade dos indivíduos. Já, o índice de BAL variou de $0,0973 \mathrm{~m}^{2}$ a $1,36 \mathrm{~m}^{2}$, com a média geral das 25 unidades amostrais de $0,7069 \mathrm{~m}^{2}$, indicando grau relativo de competição.

Cunha (2009), estudando Cedrela odorata L. na floresta amazônica, encontrou um valor médio do $\mathrm{I}_{\mathrm{GH}}$ de 0,96, indicando que as árvores encontravam-se em grau de competição e que seu crescimento está sendo
TABELA 3 Estimativa dos índices de competição das árvores amostra de Araucaria angustifolia, em Painel, SC.

TABLE 3 Estimation of competition indexes of Araucaria angustifolia sample trees, Painel, SC.

\begin{tabular}{|c|c|c|}
\hline Árvore & $\mathrm{I}_{\mathrm{GH}}$ & BAL \\
\hline 01 & I,384I & 0,2981 \\
\hline 02 & $\mathrm{I}, 2797$ & 0,7765 \\
\hline 03 & 0,4285 & 0,5387 \\
\hline 04 & $\mathrm{I}, 0762$ & 0,5115 \\
\hline 05 & 1,0195 & 0,1593 \\
\hline 06 & 0,6022 & 0,9655 \\
\hline 07 & $0,7 \mid 28$ & 0,6319 \\
\hline 08 & 0,2291 & 1,3600 \\
\hline 09 & 0,7569 & $\mathrm{I}, 2536$ \\
\hline 10 & 0,6076 & 1,1360 \\
\hline 11 & $\mathrm{I}, 2894$ & 0,8913 \\
\hline 12 & $\mathrm{I}, 5260$ & 0,5409 \\
\hline 13 & 0,8737 & 0,6842 \\
\hline 14 & 0,9532 & 0,408 I \\
\hline 15 & 0,2438 & 0,9306 \\
\hline 16 & 0,3460 & 0,6484 \\
\hline 17 & 2,3260 & 0,0973 \\
\hline 18 & $\mathrm{I}, 9554$ & 0,3425 \\
\hline 19 & 0,7856 & 0,6664 \\
\hline 20 & 0,5092 & 0,7100 \\
\hline 21 & $\mathrm{I}, 3344$ & 0,580 I \\
\hline 22 & 0,7508 & 1,0155 \\
\hline 23 & $I,|8| I$ & $0,64 I I$ \\
\hline 24 & $\mathrm{I}, 0833$ & $\mathrm{I}, 2873$ \\
\hline 25 & $\mathrm{I}, 2394$ & 0,5967 \\
\hline
\end{tabular}

$\mathrm{I}_{\mathrm{GH}}=$ índice de Glover e Holl; $\mathrm{BAL}=$ área basal das árvores maiores que a considerada.

influenciado pelas árvores vizinhas. Para o índice de BAL, encontrou valores desde zero, sem competição, até valores de 4,26, com alto grau de competição. Zanon (2007), estudando araucária na Floresta Nacional de São Francisco de Paula, RS, encontrou valor médio do $I_{G H}$ de 0,6769 e para BAL um valor médio de $0,4153 \mathrm{~m}^{2}$, indicando grau relativo de competição.

Nota-se, ainda, pelos índices de competição, que as árvores da amostra oito são as que estão em maior competição, menor $\mathrm{I}_{\mathrm{GH}}$ e maior valor de BAL, enquanto as da amostra 17 as que estão em menor competição, maior espaço para crescimento, pois apresentou o maior valor de $\mathrm{I}_{\mathrm{GH}}$ e menor valor de BAL. 


\section{CONCLUSÕES}

A floresta apresentou baixa densidade de indivíduos por hectare, caracterizando interferência antrópica com exploração em períodos passados.

○ fragmento florestal não apresentou estrutura balanceada, pois há déficit de árvores nas classes diamétricas, apresentando problemas com ingresso e regeneração.

Pela proposição de manejo com o método de Liocourt, poderiam ser retirados $86,8 \mathrm{~m}^{2}$ de área basal na área desse fragmento, para um diâmetro de manejo de $40 \mathrm{~cm}$, e 16I,76 $\mathrm{m}^{2}$ de área basal total para o diâmetro de $45 \mathrm{~cm}$.

Apesar da baixa densidade de árvores por hectare, algumas parcelas e suas árvores-amostra encontram-se em competição, justificando a intervenção com base no manejo de Liocourt.

\section{REFERÊNCIAS}

ALVES JÚNIOR, F. T.; FERREIRA, R. L. C.; SILVA, J. A. A. da; MARANGON, L. C.; COSTA JÚNIOR, R. F.; SILVA, S. de O. Utilização do quociente de De Liocourt na avaliação da distribuição diamétrica em fragmentos de Floresta Ombrófila Aberta em Pernambuco. Ciência Florestal, Santa Maria, v. 20, n. 2, p. 307-319, abr./jun. 2010.

AUBREVILLE, A. A floresta de pinho no Brasil. Anuário Brasileiro de Economia Florestal, Rio de Janeiro, v. 2, p. $21-36,1954$.

BACKES, P.; IRGANG, B. Árvores do sul: guia de identificação e reconhecimento ecológico. Porto Alegre: Pallotti, 2002. 325 p.

CAMPOS, L. J. D. Prognose por classe de diâmetro a partir de modelos do tipo povoamento total. 1997. 57 p. Dissertação (Mestrado em Ciência Florestal) Universidade Federal de Viçosa, Viçosa, 1997.

CUNHA, T. A. da. Modelagem do incremento de árvores individuais de Cedrela odorata L. na floresta amazônica. 2009. 87 f. Dissertação (Mestrado em Engenharia Florestal) - Universidade Federal de Santa Maria, Santa Maria, 2009.

DE LIOCOURT, F. De l'amenagement des sapinières. Paris: Société Forestière de Franche-Comté et Belfort, 1898.

DELLA-FLORA, J. B. Modelos de crescimento para árvores singulares - Nectandra magapotamica (Spreng) Mez. e Ocotea puberula Ness. 200I. 60 f. Dissertação (Mestrado em Engenharia Florestal) - Universidade Federal de Santa Maria, Santa Maria, 200 I.

EMPRESA DE PESQUISA AGROPECUÁRIA E EXTENSÃO RURAL DE SANTA CATARINA. Dados e informações biofísicas da Unidade de planejamento regional Planalto Sul Catarinense - UPR 3. Florianópolis, 2002. 70 p.
HESS, A. F; CALGAROTTO, A. R.; PINHEIRO, R.; WANGINIAK, T. C. R. Proposta de manejo de Araucaria angustifolia utilizando o quociente de Liocourt e análise de incremento, em propriedade rural no município de Lages, SC. Pesquisa Florestal Brasileira, Colombo, v. 30, n. 64, p. 337-345, nov./dez. 2010.

HUECK, K. As florestas da América do Sul: ecologia, composição e importância econômica. São Paulo: Polígono, 1972. $465 \mathrm{p}$.

KLEIN, R. M. O aspecto dinâmico do pinheiro brasileiro. Sellowia, Itajaí, v. 12, p. 17-44, 1960.

MACHADO, S. A.; AUGUSTYNCZIK, A. L. D.; NASCIMENTO, R. G. M.; FUGURA, M. A.; SILVA, L. C. R. da; MIGUEL, E. P.; TÉO, S. J. Distribuição diamétrica de Araucaria angustifolia (Bert.) O. Ktze. em um fragmento de floresta Ombrófila Mista. Scientia Agraria, Curitiba, v. 10, n. 2, p. 103-I I0, mar./abr. 2009.

MARTINS, F. R. Estrutura de uma floresta mesófila. Campinas: UNICAMP, 199I. 245 p.

NASCIMENTO, A. R. T.; FLEFILI, J. M.; MEIRELLES, E. M. Florística e estrutura da comunidade arbórea de um remanescente de Floresta Estacional Decidual de encosta, Monte Alegre, GO, Brasil. Acta Botanica Brasilica, São Paulo, v. I8, n. 3, p. 659-669, jul./set. 2004.

NUNES, Y. R. F.; MENDONÇA, A. V. R.; BOTEZELLI, L.; MACHADO, E. L. M.; OLIVEIRA FILHO, A. T. O. Variações da fisionomia da comunidade arbóreos em um fragmento de floresta Semidecidual em Lavras, MG. Acta Botânica Brasílica, São Paulo, v. 17, n. 2, p. 213-229, 2003.

PRODAN, M.; PETERS, R.; COX, F.; REAL, P. Mensura forestal. San José: Instituto Interamericano de Cooperación para la Agricultura, 1997. $562 \mathrm{p}$.

SCHAAF, L. B.; GALVÃO, F; SANQUETTA, C. R. Alteração na estrutura diamétrica de uma floresta ombrófila mista no período entre 1979 e 200I. Revista Árvore, Viçosa, v. 30, n. 2, p. 283-295, 2006.

SCHNEIDER, P. R. Introdução ao manejo florestal. Santa Maria: UFSM, 1993. 348 p.

SONEGO, R. C.; BACKES, A. E.; SOUZA, A. F. Descrição da estrutura de uma floresta Ombrófila Mista, RS, Brasil, utilizando estimadores não-paramétricos de riqueza e rarefação de amostras. Acta Botânica Brasílica, São Paulo, v. 21, n. 4, p. 943-955, 2007.

ZANON, M. L. B. Crescimento da Araucaria angustifolia (Bertol.) Kuntze diferenciado por dioicia. 2007. I $10 \mathrm{f}$. Tese (Doutorado em Engenharia Florestal) - Universidade Federal de Santa Maria, Santa Maria, 2007. 